\title{
Modelling the potential impacts of locational versus system-wide strike prices in contracts for difference for low carbon generation
}

\author{
Shona Pennock, Simon Gill, Keith Bell \\ University of Strathclyde \\ Glasgow, UK \\ shona.pennock@strath.ac.uk, simon.gill@strath.ac.uk, keith.bell@strath.ac.uk
}

\begin{abstract}
This paper describes the use of a cost-minimisation algorithm to explore the potential impact of two options for financial support for low carbon generation in the form of contracts for difference in a system with locational marginal pricing: 1. with a system-wide strike price; 2 . with locational strike prices. A two zone system is modelled with the additional financial support for low carbon generation represented as negative variable costs that have the effect of filling in the difference between wholesale market prices and the strike price, the latter intended to cover the long-run costs of low carbon generation. The British case is modelled in which there is a limit to the total top-up expenditure. It is shown that the case of a system-wide strike price can result in less new low carbon generation capacity compared with the case of locational strike prices, due to the increased top-up spend in the lower price zone more rapidly meeting the constraint on the total cost of top-up payments to low carbon generation. However, it is also shown that the imposition of this constraint leads to a failure of the model to settle on one solution due to the non-convex relationship between installed capacity of low carbon generation and wholesale market price.
\end{abstract}

Index Terms-power system modelling, investment planning, cost-minimisation, zonal pricing, low carbon subsides

\section{INTRODUCTION}

Investment in power systems is often modelled using optimisation software which minimise the costs over a particular time horizon [1-3]. These models require inputs such as the capital and operating costs of different types of generators and can incorporate a number of policy interventions such as additional carbon taxes which increase the variable costs of particular generators. When appropriate costs and constraints are included, these optimisations can provide useful insights into how the generation mix may develop over time.

Low carbon and renewable generation are for the most part not yet cost competitive with thermal generation such as CCGT, and are subsidized by governments to encourage investment in such projects. Modelling subsidies in costminimisation software involves making appropriate changes to the costs against which the model optimizes. In the case of subsidies paid against energy output of generators this can be achieved by subtracting the subsidy from the variable costs effectively treating it as a negative variable cost.

Subsidies to low carbon generation in Great Britain (GB) currently take the form of Feed-in-Tariffs with Contracts for Difference (CfDs) [4]. The CfD scheme involves a top-up payment added to a reference price based on the wholesale price in order that the contract holder receives an agreed strike price per MWh of generation. If the strike price is greater than the reference price, the generator is paid the difference, and if the strike price is less than the reference price, the generator has to pay the difference back. This is illustrated in Figure 1. Strike prices are allocated by competitive auction and the available spend on top-up payments is currently limited by the Levy Control Framework (LCF) budget [5].

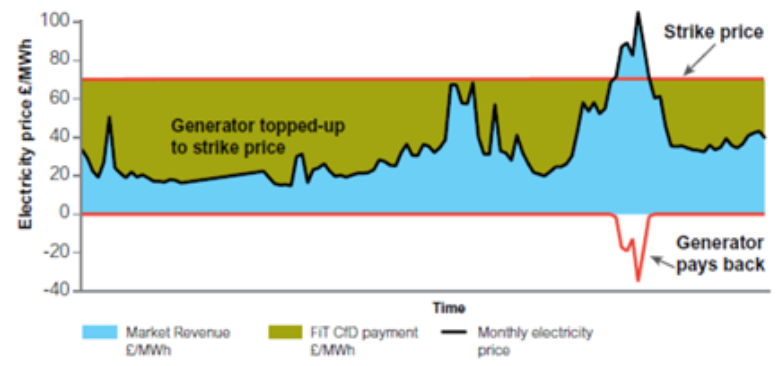

Figure 1. CfD mechanism illustration, from reference [4]

Modelling CfD subsidies in a cost-minimisation problem is more complex than modelling a straightforward feed-intariff, where a fixed payment is made per MWh generated, as CfD top-up payments to generators depend on the wholesale price of electricity which is itself an output of the optimisation. In order to set the correct value for the top-up payment each simulation needs, in effect, an estimation of what the output wholesale price will be. Assuming perfect competition, wholesale prices can be represented by the short run marginal cost (SRMC) of generation for each time period modelled. Introducing a negative cost (subsidy payment) to the variable costs of low carbon generation impacts the SRMC and thus has a feedback on the top-up payments. A solution which allows the impact of these feedback loops to be modelled in cost-minimisation software involves an iterative process where the output wholesale prices of one run 
are used to estimate the input top-up payments of the next run based on a strike price that stays fixed across iterations. The process is repeated until the output wholesale prices and input reference prices for top-up payments converge. However, convergence can only be guaranteed if a continuity and convexity condition are met, which this paper shows will not always be the case.

\section{A. Zonal pricing in $G B$}

A further complication to the modelling process is the consideration of possible multiple price zones in GB. The target model for the European electricity market has a requirement for zonal pricing for congestion management [6]. A known constraint on power transfers between Scotland and the rest of GB could warrant the establishment of Scotland as one bidding zone and England and Wales as another. How the CfD regime would adjust to consider two zonal prices within the same market, rather than a single system-wide price, is an open question. Zonal prices for Scottish generation would mostly likely be lower than those in the rest of GB due to large wind, nuclear and hydro capacities in Scotland and the low running costs of low carbon generation. These lower zonal prices would impact on CfD top-up payments, and if the government were to keep a single auction for GB-wide strike prices, a Scottish generator would use more of the LCF budget than one in England or Wales with the same strike price. This would result in less spend available for other low carbon projects.

As the overall aim of the CfD scheme is to enable enough low carbon generation to meet the UKs decarbonisation commitments at least cost to the consumer, one option would be a requirement for separate strike prices for Scottish generators in order to (1) not use up the LCF budget too rapidly; and (2) to maintain the price signals to generation from zonal pricing. The auction process would thus have to take account of the relative uplift (top-up payments) from generation in either price zone to minimize the overall LCF spend for the maximum amount of low carbon generation.

This paper explores the use of a cost-minimisation solver in the simulation of scenarios with zonal reference prices and zonal strike prices in a two zone GB system and compares the resultant installed capacity and total generation investment cost of these scenarios with a base case in which GB remains a single price zone. The modelling takes the form of a simple two node linear solver used to outline the modelling challenges in a clear and understandable framework. This solver is introduced in Section II. Section III presents the results for the zonal modelling scenarios compared with a base case scenario representing current market arrangements, Section IV discusses these results and the issues encountered and Section V draws the key conclusions from the work.

\section{REPRESENTATION OF LOW CARBON SUBSIDIES}

This section introduces a simple two node model designed to illustrate the impacts of subsidy payments and zonal pricing where the model mathematics are simple enough to easily interpret the results without additional constraints impacting upon their clarity. The model setup here results in an objective function which minimizes the total cost of energy and includes carbon pricing and the LCF as currently set up in GB.

\section{A. Mathematical description - two node model}

A two node model has been created to represent GB, in which one node represents Scotland and the other represents the rest of GB (rGB). Demand is split between the two nodes in accordance with present day demand as is modelled as increasing linearly at both nodes over a seven year timeframe. It has also been separated into a peak and off-peak block in each year. Candidate generators for investment are included as continuous variables of installed CCGT or wind at each node. Decision variables are the new installed capacity for each year and the dispatch of generation in each demand block. As demand increases in each year of the simulation, there must be an investment in some form of new generation every year in order to meet a total energy balance constraint.

The objective function minimizes the discounted cost of investment and dispatch as follows:

$$
\begin{aligned}
& \min \sum_{g, y, b} \frac{1}{(1+r)^{y-1}}\left(\text { Capex }_{g, y} \cdot \text { NIC }_{g, y}+\text { Omfix }_{g} \cdot T I C_{g, y}\right. \\
& \left.+\left(\text { Omvar }_{g, y, b}+F C_{g, y, b}+C C_{g, y, b}-S u b s_{g, y, b}\right) \cdot \text { Gen }_{g, y, b}\right)
\end{aligned}
$$

where $r$ is the discount rate, Capex $g, y$ is the capital cost associated with installing each type of generation $g$ in each year $y, N I C_{g, y}$ is the new installed capacity of each type of generation in each year, Omfix $g$ is the operation and maintenance fixed cost for generation type $g, T I C_{g, y}$ is the total installed capacity of each type of generation in each year, Omvar $_{g, b}$ is the variable operation and maintenance costs for each type of generation in each demand block $b$, $F C_{g, y, b}$ are the fuel costs associated with each type of generation in each demand block and year, $C C_{g, y, b}$ are the carbon costs associated with each type of generation in each demand block and year, $S u b s_{g, y, b}$ is the CfD subsidy (top-up payment) to each generator in each demand block and year and $G e n_{g, y, b}$ is the total generation for each generator in each demand block and year.

The following constraints are applied, representing that the generation in each block and year must not exceed each generators maximum capacity factor (2), that generation in all blocks and years must meet demand in all blocks and years (3) and that the generation at the Scottish node and rGB node in each block and year must not be greater than the node demand in each block and year plus the transfer capacity (4):

$$
\begin{gathered}
\text { Gen }_{g, y, b} \leq M C F_{g} \cdot t_{b} . T I C_{g, y} \\
\sum_{g} \text { Gen }_{g, y, b}=\operatorname{Dem}_{b, y} \\
\text { Gen }_{z, y, b} \leq \operatorname{Dem}_{z, y, b}+P T C_{y, b}
\end{gathered}
$$

where $M C F_{g}$ is the maximum capacity factor for each generator, $t_{b}$ is the time period of each block, $D e m_{y, b}$ is the 
demand in each block and year, the subscript $z$ represents each zone/node, and $P T C_{y, b}$ is the transfer capacity limit in each block and year. To represent a scenario without transmission constraints $P T C_{y, b}$ is set to a number higher than total model demand.

Capital costs are discounted using the ratio of annuity factors shown in (5) to take account of the model time horizon of seven years, assuming a generator economic lifetime of twenty-five years.

$$
\text { Capex }_{g, y}=\text { TCapex }_{g} \cdot \frac{\text { AnnAmort }}{A n n M_{y}}
$$

where TCapex $_{g}$ is the total capital expenditure, before annuity factor adjustment, and AnnAmort and $A n n M_{y}$ are the annuity factors for the amortization time and model lifetime for each generator given in (6) and (7) below:

$$
\begin{gathered}
\text { AnnAmort }=\frac{r(1+r)^{\text {amort }-1}}{(1+r)^{\text {amort }}-1} \\
\text { AnnM }_{y}=\frac{r(1+r)^{\text {yrem }-1}}{(1+r)^{\text {yrem }-1}}
\end{gathered}
$$

where amort is the amortization time for the generator (set to 25 years for all generators in this model) and yrem is the remaining model lifetime for the generator, determined by the year of installation in the model time horizon.

The CfD top-up payment is calculated as the difference between the strike price and the reference price, the latter of which is assumed to be the annual average short run marginal cost (SRMC). The SRMC is calculated as the short-run costs (fuel, carbon costs and variable operational costs, minus any subsidy) of the marginal generator for each block, time averaged. Mathematically:

$$
\begin{gathered}
S u b s_{g, y}=\text { StrikeP }_{g}-S R M C_{y} \\
S R M C_{y}=\frac{\max \left(S R C_{g, y, b 1}\right) \cdot t_{b 1}+\max \left(S R C_{g, y}, b 2\right) \cdot t_{b 2}}{\left(t_{b 1}+t_{b 2}\right)} \\
S R C_{g, y, b \#}=\text { Omvar }_{g, y, b}+F C_{g, y, b}+C C_{g, y, b}-S u b s_{g, y, b}
\end{gathered}
$$

where StrikeP $P_{g}$ is the strike price, set as the levelised cost of generation, $S R M C_{y}$ is the GB-wide SRMC in each year and $S R C_{g, y, b}$ is the short run costs of each generator in a certain demand block and year as shown in (10). As the SRMC is an output of the model, but also an input of the top-up payment calculation, the model has to be run several times and iterated until the output SRMC matches the input SRMC.

Reducing the operational costs of wind generation by use of a subsidy 'payment' makes wind generation comparatively cheaper to invest in than a CCGT. To ensure that the model invests in an appropriate amount of wind corresponding to GB policy, a representation of the LCF budget is included in the model as a constraint to the total subsidy spend, where the total top-up payments to all generators in all years must not exceed the overall LCF limit figure, as shown in (11):

$$
\sum_{g, y} S u b s_{g, y} \leq \text { LCFlimit }
$$

\section{B. Mathematical description - zonal scenarios}

To simulate zonal pricing using the two node model, the SRMC calculation has to be adapted for each zone so that in the case of transmission congestion separate zonal SRMCs are used as reference prices to calculate the zonal CfD top-up payments. When there is no congestion, the model-wide SRMC is calculated as in (9). When there is congestion the transfer capacity constraint (4) is active, and the adapted zonal SRMC calculation (12) is used, in which the zonal SRMC is calculated separately for the Scottish and rGB nodes based on the marginal generator occurring at each node.

$$
S R M C_{z, y}=\frac{\max \left(S R C_{z, g, y, b 1}\right) \cdot t_{b 1}+\max \left(S R C_{z, g, y, b 2}\right) \cdot t_{b 2}}{\left(t_{b 1}+t_{b 2}\right)}
$$

The two zonal scenarios represented are those discussed in Section I. The first includes top-up payments from a zonal reference price to a GB-wide strike price, assuming the current CfD auction system is unchanged on the introduction of zonal pricing. The second includes top-up payments to a zonal strike price, assuming CfD auction bids based on uplift, resulting in the same top-up payment to all generators no matter where they are located.

The zonal scenarios objective function is represented mathematically in (13):

$$
\begin{aligned}
& \min \sum_{z, g, y, b} \frac{1}{(1+r)^{y-1}} \text { (Capex }_{g, y} . N I C_{g, y}+\text { Omfix }_{g} . \text { TIC }_{g, y} \\
& \left.+\left(\text { Omvar }_{g, b}+F C_{g, y, b}+C C_{g, y, b}-S u b s_{z, g, y, b}\right) \cdot G e n_{z, g, y, b}\right)
\end{aligned}
$$

where $S u b s_{z, g, y, b}$ is the zonal subsidy to each generator in each block and year. Maximum capacity factor, demandmatching and transmission capacity constraints are the same as shown previously in (2)-(4).

In the case of the GB strike price scenario, the subsidy payments are shown in (14):

$$
\operatorname{Subs}_{z, g, y}=\text { StrikePGB}_{g}-S R M C_{z, y}
$$

where StrikePGB $g$ is the GB-wide strike price for wind generation. In the case of the zonal strike price scenario, the subsidy payments are shown in (15):

$$
\text { Subs }_{z, g, y}=\text { StrikeP }_{z, g}-S R M C_{z, y}
$$

where Strike $P_{z, g}$ is the zonal strike price for wind generation.

\section{Two BUS MODEL SCENARIOS AND RESULTS}

\section{A. Scenarios and model input data}

Due to the simplifications and assumptions involved, scenario comparison is the most valid way to derive insights from the two node model. The three scenarios compared in 
this paper are a base case described in Section II-A with an unconstrained network and the two zonal scenarios described in Section II-B, hereafter referred to as 'GB strike price' and 'zonal strike price'. An unconstrained base case is chosen for comparison with the zonal scenarios as it best represents the current GB system, in which decentralised trading occurs without limitations due to available transfer capacity, as system balancing occurs once trading has taken place and, through being able to submit negative bid prices into the balancing mechanism to cover the opportunity costs associated with output reduction, curtailed low carbon generation still receives market revenues and subsidy payments [7].

Data inputs to the model are shown in Table 1. Total capital cost figures prior to annuity factor discounting are shown (Total CAPEX) in addition to example values for year 1 and 7 calculated using equations (5)-(7) (Model CAPEX). Carbon and fuel prices are shown only for year 1 and 7 and follow a linear relationship over the seven years. CfD strike prices were assumed at the levelised cost of wind technology in rGB, calculated over the seven year timeframe using model input data. Maximum capacity factors were assumed to be higher for wind generation in Scotland. The availability of the generators is simply represented by these capacity factors in the annual resolution, i.e. plant closures and wind resource are not explicitly modelled. All costs are discounted to the first year of simulation using a discount rate of $6.5 \%$. Example demand values were used in Scotland and rGB, also linearly rising between years 1 and 7, with Scottish demand equal to $10 \%$ of demand in rGB.

Table 1. Data included in the two bus model

\begin{tabular}{|c|c|c|c|}
\hline & Year 1 & Year 7 & Source \\
\hline Total CAPEX wind (£/MW) & \multicolumn{2}{|c|}{$1,802,905$} & {$[8]$} \\
\hline Model CAPEX wind (£/MW) & $824,642.1$ & $141,181.3$ & [8] \\
\hline Total CAPEX CCGT (£/MW) & \multicolumn{2}{|c|}{612,370} & [8] \\
\hline Model CAPEX CCGT(£/MW) & $280,095.8$ & $47,953.28$ & {$[8]$} \\
\hline OMfix wind $(£ / M W)$ & \multicolumn{2}{|c|}{25220} & {$[8]$} \\
\hline OMfix CCGT (£/MW) & \multicolumn{2}{|c|}{42166} & {$[8]$} \\
\hline OMvar wind (£/MWh) & \multicolumn{2}{|c|}{0.105} & {$[8]$} \\
\hline OMvar CCGT (£/MWh) & \multicolumn{2}{|c|}{5.26} & [8] \\
\hline Carbon price $(£ / t \mathrm{Co} 2)$ & 18 & 50 & [9] \\
\hline Fuel price CCGT (£/MWh) & 20.58 & 24.74 & {$[10]$} \\
\hline Strike Price wind (£/MWh) & \multicolumn{2}{|c|}{104.4} & - \\
\hline Net thermal efficiency CCGT & \multicolumn{2}{|c|}{0.53} & - \\
\hline Max capacity factor CCGT & \multicolumn{2}{|c|}{0.8} & - \\
\hline Max capacity factor wind Sco & \multicolumn{2}{|c|}{0.3} & - \\
\hline Max capacity factor wind rGB & \multicolumn{2}{|c|}{0.25} & - \\
\hline Discount rate & \multicolumn{2}{|c|}{0.065} & - \\
\hline Demand rGB $(\mathrm{GW})$ & 34.2 & 47.9 & - \\
\hline Demand Scotland (GW) & 3.42 & 4.79 & - \\
\hline Net transfer capacity (GW) & \multicolumn{2}{|c|}{3.3} & - \\
\hline LCF constraint (billion $£$ ) & \multicolumn{2}{|c|}{33} & - \\
\hline
\end{tabular}

\section{B. Zonal scenarios results}

Introducing zonal scenarios to the model includes the setting of zonal subsidy payments to represent the different revenue streams available to Scottish and rGB generation in the case of market splitting, as described in Section II-B. Figure 3 shows the resultant seven-year average revenue streams of low carbon generation in the two zonal scenarios, compared with the base case scenario. It can be seen that the two zonal scenarios produce a lower average zonal price (represented by zonal SRMC) in Scotland. This is due to market splitting in some model years in addition to a higher investment in wind in Scotland (where capacity factors are higher) driving down the Scottish SRMC when market splitting takes place, i.e. when exports from Scotland reach the network transfer limit.

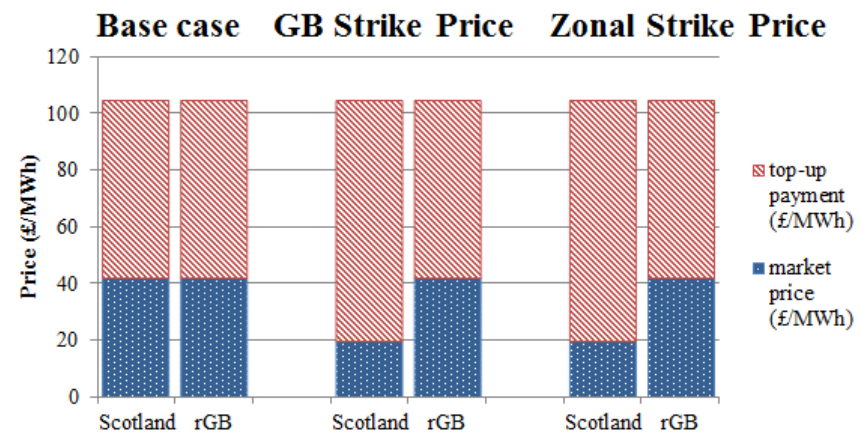

Figure 3. Revenue streams for low carbon generation for the base case and two zonal scenarios. Results for the GB strike price scenario are for case (b), described below.

Figure 4 shows the total installed renewable capacity for each model year in the base case and the two zonal scenarios. It is found that wind capacity is only built in the Scottish node in all scenarios, due to the higher Scottish capacity factor and thus lower levelised cost in this node compared with wind in rGB. In the base case, the maximum amount of wind capacity to hit the LCF constraint is invested in in the first year, after which CCGT is installed to meet the rising model demand. There is less wind installed in year 1 in the zonal scenarios compared with the base case due to the transmission constraint limiting the amount of generation able to be exported from Scotland. In the Zonal strike price scenario further wind capacity is installed in each year as Scottish demand increases, until the LCF limit is met, which shown by a change in gradient in Figure 4, e.g. after year 5 for the Zonal strike price scenario. This results in a higher installed renewable capacity in this scenario compared with the base case by year 4 .

There are two results shown for the GB strike price scenario, labelled (a) and (b). This is because the iteration process for determining the SRMC and CfD top-up payments under the LCF did not converge, resulting in two sets of results that repeat rather than converging to a single solution. The model has perfect foresight over the seven year timeframe and can install, and thus dispatch, more wind generation when top-up payments are lower, under the LCF constraint. In the case of (a), results are the same as the Zonal 
Strike Price scenario. Wind capacity is built and utilised in years 1 to 5, resulting in low Scottish SRMC's in years 1 to 4, and thus higher top-up payments in these years in the following iteration (b). In this iteration wind capacity is only built in year 1 as the higher top-up payments use up the LCF budget. This results in high Scottish SRMC's output in all years as wind generation is insufficient to cause market splitting. High SRMCs result in lower top-up payments in all years. In the next iteration, the results are the same as in (a), as the lower top-up payments allow the model to invest in more wind while still keeping within the LCF limit. This pattern repeats and does not converge to a solution. The resultant installed capacities for the GB strike price scenario (b) give a lower overall installed wind capacity than the base case, the Zonal strike price scenario and the GB strike price scenario (a). This is due to the comparatively higher top-up payments to Scottish wind generation in this scenario, as illustrated in Figure 3, using up the LCF spending limit faster than the top-up payments for the other scenarios resulting in less wind generation overall, and thus also less investment in wind capacity.

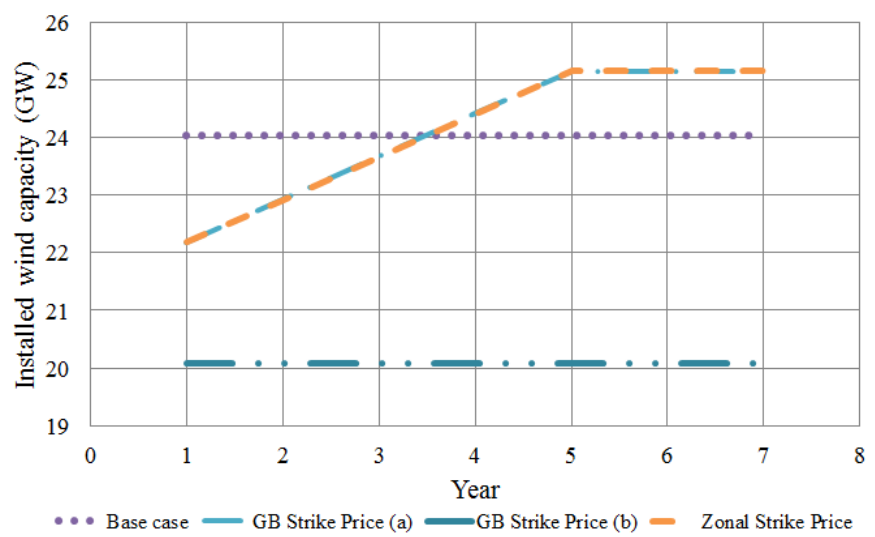

Figure 4. Installed capacity of renewable generation in the base case and each of the zonal scenarios

Table 2 shows the total generation for the base case and each of the zonal scenarios over the seven model years. It can be seen that the Zonal strike price scenario and the base case have a higher total wind generation than the GB strike price (b) results. This is due to the lower top-up payments to Scottish generation in this scenario allowing higher levels of generation under the LCF limit. This results in higher CCGT generation in the GB strike price (b) scenario to meet overall demand. The impact of this on the seven year total spend (investment in capacity, cost of generation and CfD top-ups under the LCF budget) and total carbon emissions is shown in Table 3, along with the percentage difference between the zonal cases and the base case. These figures are very similar, and the percentage differences do not exceed $3.12 \%$. A slightly higher total spend and slightly lower total carbon emissions occur in the base case and Zonal strike price scenarios than the GB strike price scenario (b) due to the higher investment and dispatch of wind.
Table 2. Total wind, CCGT and overall total generation for the zonal scenarios over the full seven year time horizon

\begin{tabular}{|c|c|c|c|}
\hline & $\begin{array}{c}\text { Total wind } \\
\text { generation } \\
(\mathrm{TWh})\end{array}$ & $\begin{array}{c}\text { Total CCGT } \\
\text { generation } \\
\text { (TWh) }\end{array}$ & $\begin{array}{c}\text { Total } \\
\text { generation } \\
\text { (TWh) }\end{array}$ \\
\hline Base case & 442.18 & 2329.82 & 2772.00 \\
\hline $\begin{array}{c}\text { Zonal strike } \\
\text { price }\end{array}$ & 443.12 & 2328.88 & 2772.00 \\
\hline $\begin{array}{c}\text { GB strike } \\
\text { price (a) }\end{array}$ & 443.12 & 2328.88 & 2772.00 \\
\hline $\begin{array}{c}\text { GB strike } \\
\text { price (b) }\end{array}$ & 369.38 & 2402.62 & 2772.00 \\
\hline
\end{tabular}

Table 3. Total spend and carbon emissions for zonal scenarios over the full seven year time horizon

\begin{tabular}{|c|c|c|c|c|}
\hline & $\begin{array}{c}\text { Total } \\
\text { spend } \\
\text { (billionf) }\end{array}$ & $\begin{array}{c}\text { Percentage } \\
\text { difference } \\
\text { with base } \\
\text { case }\end{array}$ & $\begin{array}{c}\text { Total } \\
\text { carbon } \\
\text { emissions } \\
\text { (mtCo2) }\end{array}$ & $\begin{array}{c}\text { Percentage } \\
\text { difference } \\
\text { with base } \\
\text { case }\end{array}$ \\
\hline Base case & 123.33 & - & 472.44 & - \\
\hline $\begin{array}{c}\text { Zonal } \\
\text { strike price }\end{array}$ & 123.38 & $0.04 \%$ & 472.25 & $-0.04 \%$ \\
\hline $\begin{array}{c}\text { GB strike } \\
\text { price (a) }\end{array}$ & 123.38 & $0.04 \%$ & 472.25 & $-0.04 \%$ \\
\hline $\begin{array}{c}\text { GB strike } \\
\text { price (b) }\end{array}$ & 120.54 & $-2.26 \%$ & 487.20 & $3.12 \%$ \\
\hline
\end{tabular}

IV. DISCUSSION

The results in Section III have shown a lower zonal price occurring at the Scottish node in the zonal scenarios due to market splitting and the high proportion of renewables in Scotland. This leads to a higher top-up payment to Scottish generation in the GB strike price scenario (b) when compared with the base case or Zonal strike price scenarios. Less renewable capacity is able to be installed and dispatched in the GB strike price scenario (b) due to these higher top-up payments using up the LCF limit. This scenario is slightly cheaper overall, but also produces slightly higher carbon emissions.

The results in Section III have also revealed a case in which the SRMC and top-up payment iterative calculation does not converge. This is due to the interaction between the LCF limit and the CfD mechanism in this cost-minimisation model. The CfD top-up payment is calculated using the zonal SRMC as the reference price, and the calculation of zonal SRMC is based on the amount of wind generation in each zone when a transmission constraint is present. As the total top-up is limited by the LCF constraint, a feedback loop has been created that installs wind capacity where SRMCs are high and top-up payments are low to meet the LCF limit, which in turn lowers the SRMCs and raises the top-up payments, resulting in a scenario where convergence is not possible.

Figure 5 illustrates the issue preventing the GB strike price scenario from converging. When wind penetration at the Scotland node is greater than $86 \%$, wind generation becomes the price maker for the off-peak block, meaning that the 
annual SRMC drops to almost zero. As shown in the graph, the SRMC can only take one of two values in each year, depending on if CCGT or wind is setting the price. The relationship between installed capacity of wind and SRMC is non-convex. Altering the length of the off-peak block would result in this drop in SRMC occurring at a different percentage installed wind capacity.

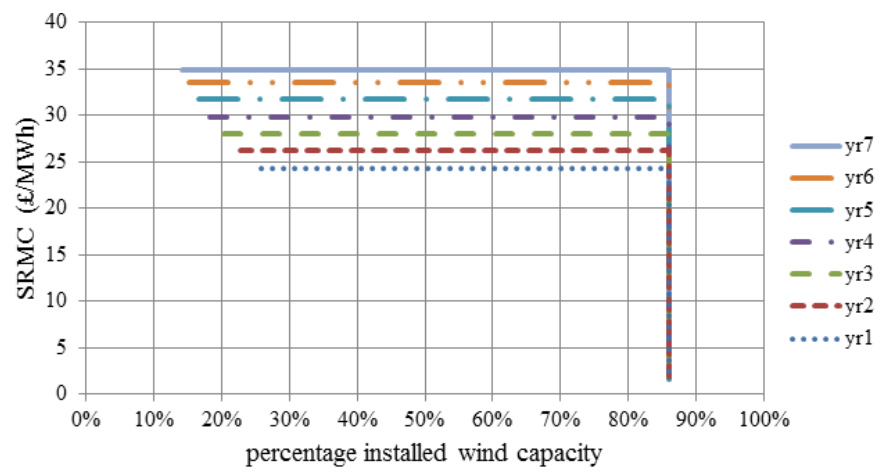

Figure 5. SRMC vs percentage installed wind capacity

The GB strike price scenario revealed this modelling issue as the only scenario with differing top-up payments dependent on zone. The Zonal strike price scenario and base case both have equal top-up payments to low carbon generation in both zones, which equally impact the LCF constraint, so convergence was not impacted by market splitting.

This modelling highlights the issues associated with using a cost-minimisation algorithm with CfD subsidies included as negative variable cost terms. In the case of CfDs the payments are a function of the wholesale electricity price, represented here by the SRMC, which adds an additional layer of complexity to the modelling with the iterative process required to ensure that input and output SRMCs converge.

The scenarios modelled here represent a case in which the cost of energy (with current low carbon generation incentives) is minimised, assuming perfect foresight and perfect competition. Further work could involve representing the problem as a profit-maximisation rather than a costminimisation, including a representation of wholesale market revenues into the objective function, to represent the relative overall revenues to generators in the zonal scenarios. These zonal scenarios need to be represented with wholesale market revenue payments as well as CfD top-up payments to illustrate the impact of zonal pricing on the operational costs, and thus lifetime costs, of all generators. Without including revenue payments the GB strike price scenario includes higher revenues (top-up payments) for Scottish generators, when in reality overall revenues (market revenues + top-up) are the same for generators in both zones. Not including revenue payments also results in the Zonal strike price scenario providing the same revenues (top-up payments) to generators in each zone, when overall revenues (market revenues + top-up) are less for generation in Scotland. The inclusion of revenues involves another negative variable cost term for all generators, and essentially changes the nature of the problem from cost-minimisation to profit-maximisation as the overall generator costs can become negative.

\section{CONCLUSIONS}

A two-node model has been described with an objective function that is a minimisation of the sum of operational and capital costs associated with investment and dispatch of generation, subject to various constraints including transmission capacity and the LCF budget. A simple model has deliberately been used to illustrate the issues with modelling CfD subsidies within a budget constraint. It has been shown that the introduction of locational marginal pricing whilst maintaining a system-wide strike price can result in a lower overall investment and dispatch of low carbon generation when compared with a case without locational marginal pricing and a case with locational marginal pricing and locational strike prices. These results suggest that if locational marginal pricing was introduced to GB, maintaining a system-wide strike price would not be the most cost-efficient solution to meet decarbonisation targets. However, it has also been shown that not all scenarios converge to a single solution when using an iterative process to calculate SRMCs and thus CfD top-up payments within the LCF constraint. It is concluded that the relationship between installed capacity of low carbon generation and wholesale market price is non-convex, something that should be addressed in specific model developments.

\section{ACKNOWLEDGMENT}

The first author acknowledges financial support from the EPSRC through the CDT in Wind Energy Systems (grant EP/L016680/1). The second author acknowledges support from ClimateXChange http://www.climatexchange.org.uk/

\section{REFERENCES}

[1] AF-Mercados, "Powerful Targets: Exploring the relative cost of meeting decarbonisation and renewables targets in the British power sector," 2012

[2] J. Richter, "DIMENSION - A Dispatch and Investment Model for European Electricity Markets," EWI working paper, 2011

[3] K. Neuhoff et al, "Space and time: wind in an investment planning model," Energy Economics 30, 2008, pp1990-2008

[4] Department of Energy and Climate Change, "Planning our electric future: a White Paper for secure, affordable and low-carbon electricity," July 2011.

[5] National Audit Office, "The Levy Control Framework," 27 Nov 2013. Available: https://www.nao.org.uk/press-releases/levy-controlframework/

[6] ACER, "Framework Guidelines on Capacity Allocation and Congestion Management for Electricity," 29 July 2011

[7] Elexxon, "The Electricity Trading Arrangements, A Beginner's Guide," 5 November 2015. Available: https://www.elexon.co.uk/wpcontent/uploads/2015/10/beginners_guide_to_trading_arrangements _v5.0.pdf

[8] Department of Energy and Climate Change, "Electricity Generation Costs," December 2013. Available: https://www.gov.uk/government/ publications/decc-electricity-generation-costs-2013

[9] Committee on Climate Change, "The Fourth Carbon Budget reducing emissions through the 2020s," December 2010

[10] Department of Energy and Climate Change, "Updated energy and emissions projections: 2014" 25 September 2014. 
